This item was submitted to Loughborough's Research Repository by the author.

Items in Figshare are protected by copyright, with all rights reserved, unless otherwise indicated.

\title{
Enhanced harmonic generation at terahertz frequencies with a two- frequency wiggler
}

PLEASE CITE THE PUBLISHED VERSION

PUBLISHER

(c) IEEE

VERSION

VoR (Version of Record)

LICENCE

CC BY-NC-ND 4.0

\section{REPOSITORY RECORD}

Kong, Michael G.. 2019. "Enhanced Harmonic Generation at Terahertz Frequencies with a Two-frequency Wiggler". figshare. https://hdl.handle.net/2134/5188. 
This item was submitted to Loughborough's Institutional Repository (https://dspace.lboro.ac.uk/) by the author and is made available under the following Creative Commons Licence conditions.

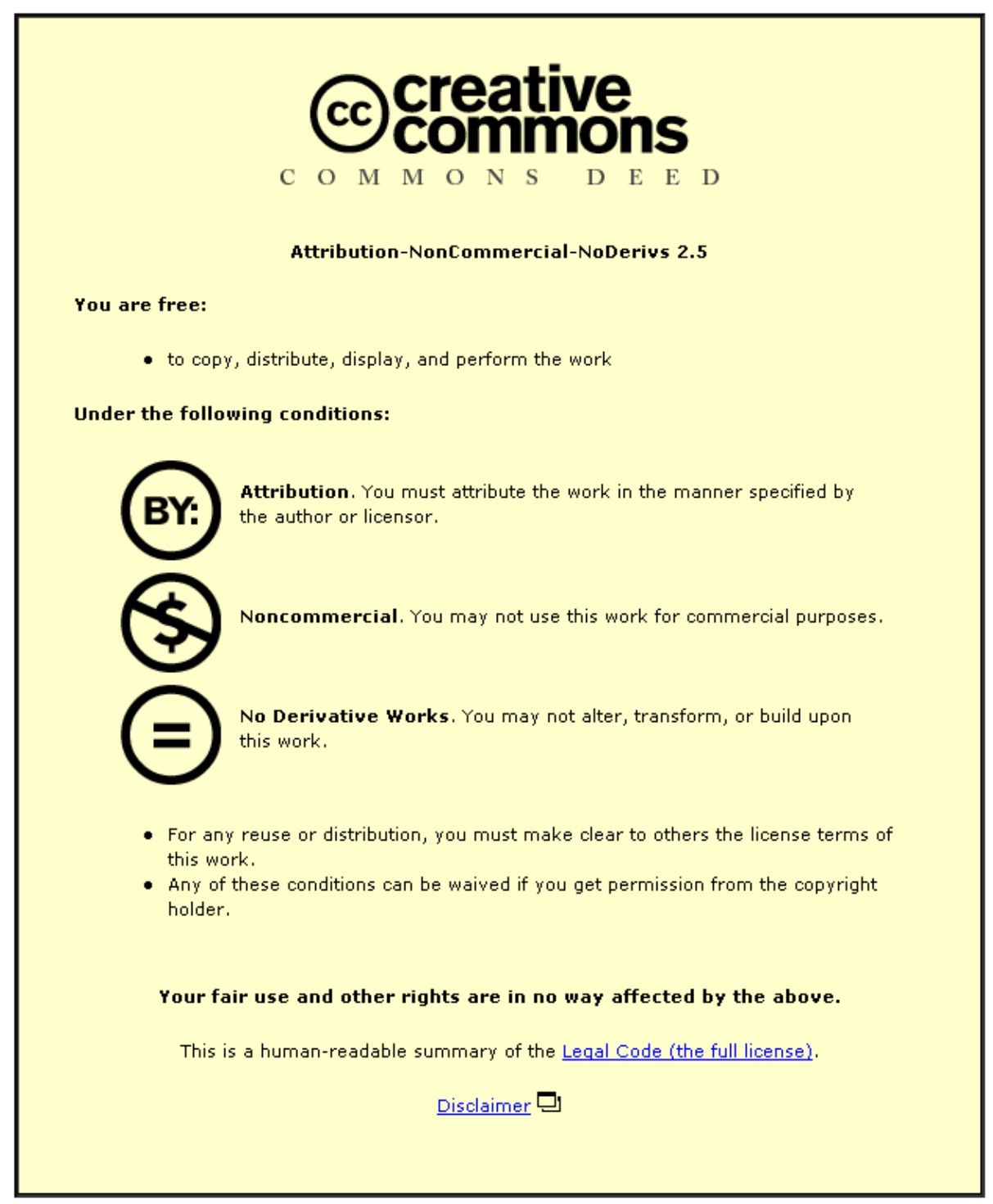

For the full text of this licence, please go to: http://creativecommons.org/licenses/by-nc-nd/2.5/ 


\title{
Enhanced Harmonic Generation at Terahertz Frequencies with a Two-Frequency Wiggler
}

\author{
Michaẹl G Kong \\ Department of Electrical Engineering and Electronics, University of Liverpool, Liverpool L69 3GJ, UK
}

\begin{abstract}
It is known that a strong harmonic radiation from free electron laser (FEL) devices could lead to a significant reduction in the minimum electron energy required to generate radiation at a given frequency. However practical application of this technique to terahertz FELs has been hammered by the very weak strength of FEL radiation at high harmonics. In this paper, a two-frequency wiggler configuration is considered as a means to enhance the strength of FEL emission at high harmonics. It is shown that by adjusting the nonlinear interference between the two constituent components of the two-frequency wiggler the emission strength around the 10th harmonic can be increased considerably by two orders of magnitude. This characteristics can then be used to reduce the electron energy needed for terahertz radiation from $2.5 \mathrm{MeV}$ to below $500 \mathrm{keV}$, thus reducing significantly size and cost of the eventual terahertz FEL system.
\end{abstract}

\section{INTRODUCTION}

The development of terahertz electronics has recently commanded much attention, largely because of its potential to aid significant technological advance in many closely related disciplines such as remote sensing, semiconductor nanostructures, communications, and time-resolved spectroscopy[1]-[4]. One important issue in the field is to develop powerful, coherent, and tunable sources at terahertz frequencies and at present free electron lasers (FELs) are perhaps one of very few such sources. Characteristics commonly associated with free electron lasers are however large size, high cost, and complex designs. Although these characteristics are acceptable for an instrument built on the scale of a national laboratory, they preclude the acquisition of such an instrument by a single university department or research group. As a result, the benefit of the FEL to the progress of terahertz technology is limited. To relieve this limitation, new FEL concepts and interaction mechanisms must be derived so as to reduce its size and cost while maintaining its more desirable features such as tunability, coherence, and large power output.

Many of the FEL's undesirable features stem from the need to employ high energy electron beams which are usually produced by bulky and expensive accelerator systems. This may be illustrated by the dependence of the radiation wavelength, $\lambda_{r}$, upon the period of the wiggler magnet, $\lambda_{w}$, and the electron energy[5]

$$
\lambda_{r}=\frac{\lambda_{w}}{2 \gamma^{2}}
$$

E-mail: mgk@liv.ac.uk and facsimile: +441517944540

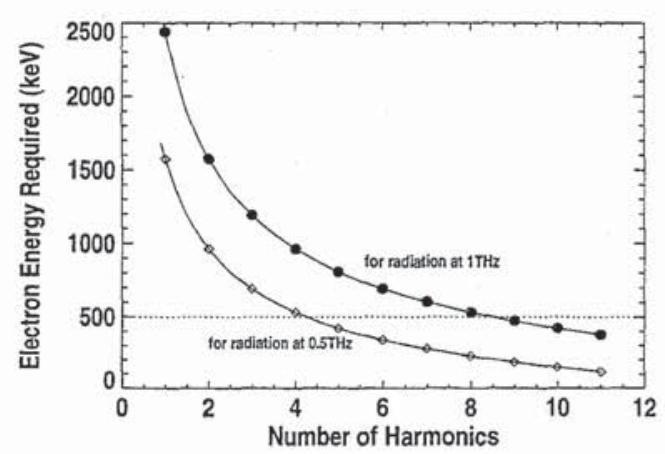

Fig. 1. Reduction in the minimum electron energy required to generate terahertz FEL radiation by operating FEL at harmonics with a single wiggler magnet of $\lambda_{w}=2 \mathrm{~cm}$.

where $\gamma$ is the relativistic factor. Suppose that quasioptical resonant cavities are used to confine FEL radiation generated at terahertz frequencies. The radiation frequency, $f$, can then be calculated from the approximation of $f=c / \lambda_{r}$. We further assume that the permanent magnet technology is employed for wiggler design and construction such that the wiggler field strength can achieve unity at periodicities of a few centimetres. Thus with a standard wiggler magnet with a period of $2 \mathrm{~cm}$, radiation at $1 \mathrm{THz}$ would require an electron beam of $2.5 \mathrm{MeV}$. With the present accelerator technology, electron beams of $2.5 \mathrm{MeV}$ are likely to be produced by either electrostatic accelerators or induction linacs, both of which are of large size and thus high cost[6]. One way to reduce the minimum electron energy required to generate radiation at a given frequency is to operate the FEL at its harmonics and this is best understood by examining the FEL resonant condition[5]

$$
\lambda_{r}=\frac{\lambda_{w}}{2 h \gamma^{2}}
$$

where $h$ is the number of harmonics. Figure 1 shows that by operating the FEL at its harmonics how the minimum necessary electron energy for generating radiation at terahertz frequencies is reduced when $\lambda_{w}=2 \mathrm{~cm}$.

In a conventional FEL system with a planar wiggler magnet, harmonic generation originates from a harmonic oscillation of the axial electron velocity and the emission strength decreases as the harmonic number increases. Thus although significant reduction in electron energy may be achieved by harmonic operation, an effective application of this technique is hammered by the considerably lower radiation strength at high harmonics than that at the funda- 


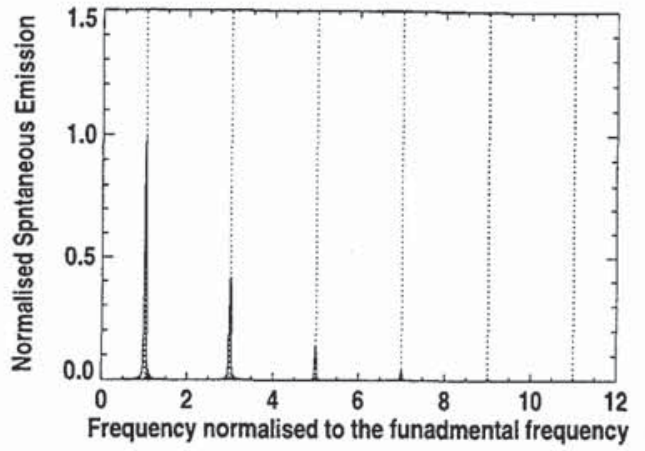

Fig. 2. Spontaneous emission spectrum of a conventional FEL

mental frequency. As a result, the eventual system may operate below the threshold for substantial oscillation at harmonic frequencies. To illustrate this more clearly, we consider the spontaneous emission from a conventional FEL with a planar wiggler. Based on the formulation developed in [5], the FEL spontaneous emission normalised to its peak value at the fundamental frequency is plotted in figure 2 as a function of the radiation frequency. Figure 2 shows that as the harmonic number increases the FEL radiation decreases. In particular, the emission strengths at the 9 th and 11th harmonics are $0.97 \%$ and $0.23 \%$ of that at the fundamental frequency, respectively. Thus for an effective usage of FEL harmonic radiation, its spontaneous emission strength at high harmonics needs to be enhanced considerably.

In this paper, we consider possible enhancement of FEL harmonic generation with a two-frequency wiggler configuration that consists of two conventional wigglers of different periods. Harmonic generation in such an unconventional wiggler configuration is based on a nonlinear interference effect rather than the harmonic oscillation in the axial electron velocity. This work is motivated by recent work on manipulation of FEL gain and spectrum by means of unconventional wiggler configurations[7]-[10].

\section{Spontaneous Emission Formulation}

Consider a magnet system composed of two different conventional planar wigglers of periods $\lambda_{w 1}$ and $\lambda_{w 2}$, respectively. For the simplicity, we assume that $\lambda_{w 1}>\lambda_{w 2}$ and that the on-axis magnetic field of this two-frequency wiggler configuration may be approximated as

$$
\vec{B}_{w}=\hat{y}\left(B_{w 1} \cos k_{w 1} z+B_{w 2} \cos k_{w 2} z\right)
$$

where $k_{w n}=2 \pi / \lambda_{w n}(n=1,2)$. Suppose the lengths of the two constituent wigglers are $L_{1}=N_{1} \lambda_{w 1}$ and $L_{2}=N_{2} \lambda_{w 2}$, respectively. Without violating generality, we assume $L_{2}>$ $L_{1}$ and $N_{2}=1+\operatorname{Int}\left[L_{1} / \lambda_{w 2}\right]$. Therefore eq.(1) is valid for $0<z \leq L_{1}$, whereas in the small region of $L_{1}<z \leq L_{2}$ the wiggler field is $\vec{B}_{w}=\hat{y} B_{w 2} \cos k_{w 2} z$.

Under the perturbation of the alternating magnetic field of eq.(1), an energetic electron beam travelling along the wiggler axis radiates and the induced electromagnetic radi- ation may be represented by its on-axis electric field component of

$$
\vec{E}=\hat{x} E_{0} \sin \Phi=\hat{x} E_{0} \sin (\omega t-k z+\phi) .
$$

The trajectory of an electron beam in the combined field of the wiggler magnet and the induced radiation is in general governed by its equation of motion

$$
\frac{d(\gamma m \vec{v})}{d t}=-e\left(\vec{E}+\vec{v} \times \vec{B}_{w}\right) .
$$

In the limit of small radiation field, the transverse velocity of an electron entering the two-frequency wiggler along the magnet axis may be easily obtained from eq.(3) as

$$
\beta_{x}=\frac{1}{\gamma}\left(a_{w 1} \sin k_{w 1} z+a_{w 2} \sin k_{w 2} z\right)
$$

where $a_{w n}=e B_{w n} / m c k_{w n}$ is the dimensionless field strength of the $n$th constituent wiggler $(n=1,2)$. Once again with the small radiation field assumption, the electron energy change may be considered to be negligible in the calculation of electron trajectory and as such the axial electron velocity can be derived from $\beta_{0}^{2}=\beta_{x}^{2}+\beta_{z}^{2}\left(\beta_{0} c\right.$ being the initial electron velocity) as

$$
\beta_{z}=\left[1-\frac{1+\left(a_{w 1} \sin k_{w 1} z+a_{w 2} \sin k_{w 2} z\right)^{2}}{\gamma^{2}}\right]^{1 / 2} .
$$

For most short wavelength free electron lasers driven by rf linacs and electron storage rings, the electron energy is highly relativistic[6]. Thus $\gamma^{2} \gg 1$ and the above equation may be approximated as

$$
\begin{aligned}
\beta_{z} \approx 1 & -\frac{1}{4 \gamma^{2}}\left\{\left(1+a_{w 1}^{2}+a_{w 2}^{2}\right)-a_{w 1}^{2} \cos 2 k_{w 1} z\right. \\
& \left.-a_{w 2}^{2} \cos 2 k_{w 2} z+2 a_{w 1} a_{w 2} \sin k_{w 1} z \sin k_{w 2} z\right\} .
\end{aligned} .
$$

It is seen that due to the nonlinear dependence of $\beta_{z}$ on the field strengths of the two wiggler components in eq.(5) the axial electron velocity contains not only terms due to individual wiggler components, $a_{w n}^{2} \cos 2 k_{w n} z(n=1,2)$, but also their cross term, $2 a_{w 1} a_{w 2} \sin k_{w 1} z \sin k_{w 2} z$. Such a cross term represents a nonlinear interference effect between the two wiggler components on the axial electron velocity. As it will be shown in section III, this nonlinear interference effect plays a very significant role in determining the FEL spectrum and thus the FEL emission at harmonics. Note that the high order terms neglected in deriving eq.(6) contain many other cross terms which have small amplitudes for highly relativistic electron beams.

The energy exchange of an electron with its radiation field is governed by the energy conservation law

$$
\frac{d\left(\gamma m c^{2}\right)}{d t}=-e \vec{E} \cdot \vec{v} .
$$

Substitution of eq.(2) into the above equation gives

$$
\begin{aligned}
\frac{d \gamma}{d t}=-\frac{e E_{0}}{2 \gamma m c} \sum_{n=1}^{2} a_{w n} & {\left[\cos \left(\Phi-k_{w n} z\right)\right.} \\
& \left.-\cos \left(\Phi+k_{w n} z\right)\right] .
\end{aligned}
$$


As illustrated in eq.(8), the axial electron velocity of eq.(6) affects crucially the beam-wave interaction described by eq.(7) via the phase angles of $\Phi \mp k_{w n} z=\omega t-\left(k \pm k_{w n}\right) z+$ $\phi$ in which the electron spatial location, $z$, needs to be expressed in terms of $t$. To this end, the electron average velocity in the axial direction, $\beta_{z 0} c$, needs to be formulated first. Integration of eq.(6) gives the average axial velocity

$$
\beta_{z 0} \approx 1-\frac{1}{2 \gamma^{2}}\left(1+\frac{1}{2} a_{w 1}^{2}+\frac{1}{2} a_{w 2}^{2}+\xi a_{w 1} a_{w 2}\right)
$$

where

$$
\xi=\frac{\sin \left(2 \pi N_{1} \lambda_{w 1} / \lambda_{w 2}\right)}{N_{1} \pi\left(\lambda_{w 1}^{2} / \lambda_{w 2}^{2}-1\right)} .
$$

Note that $\xi=1$ when $\lambda_{w 1}=\lambda_{w 2}$. However $\xi \ll 1$ if the two wiggler periods differ from each other appreciably. With $\beta_{z 0}$ formulated in eq.(9), eq.(6) becomes

$$
\begin{aligned}
\beta_{z} & =\beta_{z 0}+\frac{1}{4 \gamma^{2}}\left[a_{w 1}^{2} \cos 2 k_{w 1} z+a_{w 2}^{2} \cos 2 k_{w 2} z\right] \\
& +\frac{a_{w 1} a_{w 2}}{2 \gamma^{2}}\left[\xi+\cos \left(k_{w 1}+k_{w 2}\right) z-\cos \left(k_{w 1}-k_{w 2}\right) z\right]
\end{aligned}
$$

If we approximate $z$ with the nominal electron position $z_{0}=c \beta_{z 0} t$ on the right hand side of the above equation, a straightforward integration of $\beta_{z}$ in eq.(11) leads to the formulation of the electron spatial location as

$$
z=c \int_{0}^{z_{0} / \beta_{z 0 c}} \beta_{z}(\tau) d \tau=z_{0}+\frac{1}{k}\left[p\left(z_{0}\right)+q\left(z_{0}\right)\right]
$$

where

$$
\begin{aligned}
p\left(z_{0}\right)= & \alpha_{0} \sum_{n=1}^{2} \frac{a_{w n}^{2}}{a_{w 1} a_{w 2}} \frac{\lambda_{w n}}{\lambda_{w 1}} \sin 2 k_{w n} z_{0} \\
q\left(z_{0}\right)= & 4 \alpha_{0}\left[\xi k_{w 1} z_{0}+\frac{\sin \left(k_{w 1}+k_{w 2}\right) z_{0}}{1+\lambda_{w 1} / \lambda_{w 2}}\right. \\
& \left.\quad-\frac{\sin \left(k_{w 1}-k_{w 2}\right) z_{0}}{1-\lambda_{w 1} / \lambda_{w 2}}\right] \\
\alpha_{0}= & \frac{k a_{w 1} a_{w 2}}{8 \gamma^{2} \beta_{z 0} k_{w 1}}
\end{aligned}
$$

The terms $p\left(z_{0}\right)$ and $q\left(z_{0}\right)$ are related to the nonlinear interference between the two wiggler components of the two frequency wiggler structure, and as it will be shown in section III they affect crucially the FEL spectrum. Using eq.(12), the phase angles in eq.(8) become

$$
\begin{aligned}
\Phi \mp k_{w n} z & \approx\left[\frac{\omega}{c \beta_{z 0}}-\left(k \pm k_{w n}\right)\right] z_{0}+\phi-\left(p\left(z_{0}\right)+q\left(z_{0}\right)\right) \\
& =\Delta k_{n}^{ \pm} z_{0}+\phi-\left(p\left(z_{0}\right)+q\left(z_{0}\right)\right)
\end{aligned}
$$

where $\Delta k_{n}^{ \pm} z=\left(\omega /\left(c \beta_{z 0}\right)-\left(k \pm k_{w n}\right)\right) z$ and the approximation of $\left(k \pm k_{w n}\right) / k \approx 1$ has been assumed.

In eq.(14), all phase angles of relevance to eq.(8) are expressed in terms of the nominal electron position only. This allows eq.(8) to be integrated directly to give the electron energy change over the entire length of the two-frequency

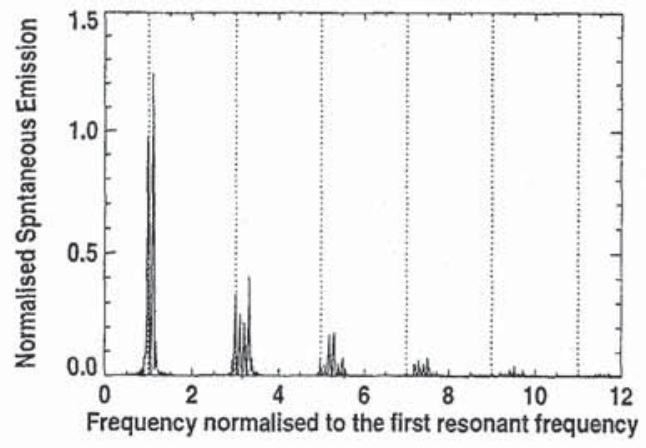

Fig. 3. FEL spontaneous emission in a two-frequency wiggler with $a_{w 1}=a_{w 2}=1$ and $\lambda_{w 1}=1.1 \lambda_{w 2}$

wiggler, $\Delta \gamma$. According to Madey's theorem on the other hand, the spontaneous emission of the electron beam is proportional to $\left\langle(\Delta \gamma)^{2}\right\rangle$ where $\langle\ldots\rangle$ denotes to an average over the initial electron phase. In the SI units, this relationship is given by[11], [12]

$$
\frac{d^{2} W}{d \omega d \Omega}=\frac{m^{2} c \omega^{2}}{8 \pi^{2} \epsilon_{0} E_{0}^{2}}<(\Delta \gamma)^{2}>.
$$

The formulation of $\left\langle(\Delta \gamma)^{2}\right\rangle$ from eqs.(8) and (14) is rather lengthy but nevertheless straightforward. It can be shown that in the limit of small radiation field the spontaneous emission is given by[12], [13]

$$
\frac{d^{2} W}{d \omega d \Omega}=\frac{a_{w 1}^{2}}{16} \sqrt{\frac{\mu_{0}}{\epsilon_{0}}}\left[\frac{e N_{1}}{\gamma\left(1-\beta_{z 0}\right)}\right]^{2} \frac{L^{2}}{L_{1}^{2}} \mathcal{S}
$$

where $a_{w n}=e B_{w n} / m c k_{w n}$ is the dimensionless field strength of the $n$th constituent wiggler $(n=1,2)$ and

$$
\begin{aligned}
\mathcal{S}= & \frac{1}{a_{w 1}^{2}} \frac{\omega^{2}}{\omega_{r 1}^{2}}\left\{\left[\sum a_{w n}\left(a_{n}^{+}-a_{n}^{-}\right)\right]^{2}\right. \\
& \left.\quad+\left[\sum a_{w n}\left(b_{n}^{+}-b_{n}^{-}\right)\right]^{2}\right\} \\
a_{n}^{ \pm}= & \frac{1}{L} \int_{0}^{L} \cos \left(\Delta k_{n}^{ \pm} z-p(z)-q(z)\right) d z \\
b_{n}^{ \pm}= & \frac{1}{L} \int_{0}^{L} \sin \left(\Delta k_{n}^{ \pm} z-p(z)-q(z)\right) d z \\
\omega_{r 1}= & \frac{k_{w 1} c \beta_{z 0}}{\left(1-\beta_{z 0}\right)} .
\end{aligned}
$$

The above expressions will be used to explore possible enhancement of harmonic radiation from free electron lasers in the next section.

\section{Application}

To illustrate the characteristics of the two-frequency wiggler configuration, we consider a two-frequency wiggler with $a_{w 1}=a_{w 2}=1$ and $\lambda_{w 1}=1.1 \lambda_{w 2}$. As it is shown in figure 3 , the addition of the second wiggler increases appreciably radiation strength at harmonic frequencies. In 


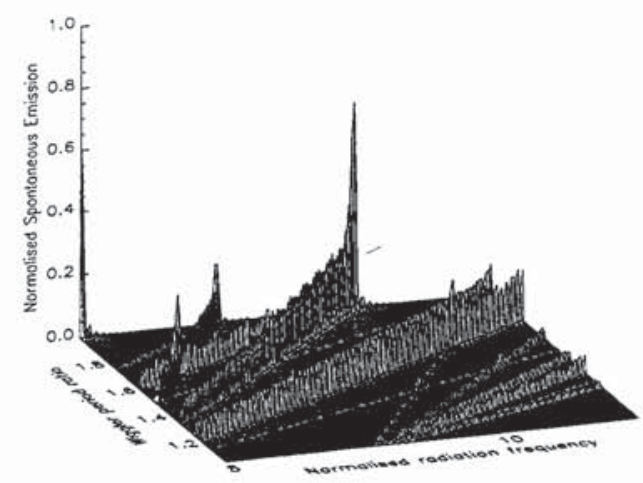

Fig. 4. FEL spontaneous emission in a two-frequency wiggler with $a_{w 1}=a_{w 2}=1$ at different wiggler period ratios

particular, detailed calculation shows that the spontaneous emission at $\omega=9.2 \omega_{r 1}$ is almost twice as much as that in the single wiggler case. This enhancement in spontaneous emission is most probably due to the nonlinear interference between the two constituent wiggler components of the two-frequency wiggler. To further explore this hypothesis, we calculate the spontaneous emission for different values of the wiggler period ratio, $\lambda_{w 1} / \lambda_{w 2}$. As shown in Figure 4 , the strength and distribution of the spontaneous emission is indeed strongly dependent upon the periodicities of the two wiggler components and consequently their interference. This finding is consistent with the results of recent work on quasiperiodic wigglers[9], [13]. More importantly, the magnitude of spontaneous emission is increased significantly. In the case of $\lambda_{w 2}=0.5 \lambda_{w 1}$, the value of the spontaneous emission around $\omega=10 \omega_{r 1}$ is now $66 \%$ of that of the fundamental spontaneous emission in a conventional FEL. Note that in a conventional FEL with the planar wiggler configuration there is no radiation at even harmonics[5], [6], but its emission strength around the 10th harmonic may be phenomenologically represented by the average of the emission strengths at the 9th and the 11th harmonics. From Figure 1, this representation would give the spontaneous emission at the 10th harmonic a phenomenological value of $(0.97 \%+0.23 \%) / 2=0.60 \%$. For the FEL with a twofrequency wiggler of $\lambda_{w 2}=0.5 \lambda_{w 1}$ in the aforementioned example, its normalised emission strength at the 10 th harmonic is $66 \%$ as suggested in Figure 4 and this represents an emission strength enhancement of $66 \% / 0.60 \%=110$ folds for FEL radiation at frequencies between the 9 th and 10th harmonics.

The above example demonstrates that with the twofrequency wiggler configuration the FEL emission at high harmonics can be made sufficiently strong to yield a similar level of power output to that in a conventional FEL operating at the fundamental frequency. This enhancement of harmonic radiation would allow FEL systems with a twofrequency wiggler to be operated at high harmonics while maintaining a level of power output adequate for many practical applications. These findings have significant im- plication in terms of the characteristics of the eventual FEL system since the electron energy needed to generate FEL radiation at terahertz frequencies can now be realistically reduced at relatively little expense of the resultant radiation strength. For instance, the electron energy for FEL radiation at $1 \mathrm{THz}$ can be reduced from $2.5 \mathrm{MeV}$ to around $422 \mathrm{keV}$ (see Figure 1) when the FEL with a two-frequency wiggler is operated around its 10 th harmonic with its radiation strength reduced by only $34 \%$ from that at the fundamental frequency.

\section{CONCLUSION}

A two-frequency wiggler configuration has been considered as a means to enhance the harmonic radiation in free electron laser devices. The spontaneous emission has been formulated, and based on this formulation numerical examples have been used to illustrate the characteristics of twofrequency wiggler based FELs. It has been shown that by adjusting the nonlinear interference between the two constituent wiggler components of a two-frequency wiggler the FEL spontaneous emission can be significantly enhanced at high harmonics. This permits the possibility of achieving an efficient FEL operation at terahertz frequencies with an electron beam energy kept below $500 \mathrm{keV}$. The generation of a strong FEL radiation at terahertz frequencies by low energy electron beams of less than $500 \mathrm{keV}$ suggests an interesting prospect of developing and building compact terahertz FELs for use in a university laboratory.

\section{REFERENCES}

[1] C. M. Snowden and D. P. Steenson, "Circuits and simulations at $1 \mathrm{THz}$ ", Phil. Trans. R. Soc. Lond. A, vol.354, pp.2435-2446, 1996.

[2] N. J. Cronin, "Terahertz systems: the demands on devices", Phil. Trans. R. Soc. Lond. A, vol.354, pp.2425-2434, 1996.

[3] S. Zeuner, S. J. Allen, K. D. Maranowski, and A. C. Gossard, "Photon-assisted tunnelling in GaAs/AlGaAs superlattices up to room temperature", Appl. Phys. Lett, vol.69, pp.2689-2691, 1996.

[4] P. L. Marston, "A time-resolved glimpse of the terahertz glory", Nature, vol.391, no.6670, pp.841-842, 1998.

[5] W. B. Colson, "The nonlinear wave equation for higher harmonics in free-electron lasers", IEEE J. Quan. Electron., vol.17, pp.1417-1426, 1981.

[6] H. P. Fruend and T. M. Antonsen, Jr, "Principles of free-electron lasers", Chapman \& Hall, London, 1992.

[7] D. Iracane and P. Bamas, "Two-frequency wiggler for better control of free-electron laser dynamics", Phys. Rev. Lett. vol.67, pp.3086-3089, 1991.

[8] M. G. Kong, Phys. Rev. E, "Enhanced optical klystron gain with a multiharmonic wiggler", vol.52, pp.3060-3065, 1995.

[9] M. Takao, S. Sasaki, and S. Hashimoto, "Spectrum formula of the synchrotron-radiation from a quasi-periodic undulator", Phys. Rev. E, vol.52, pp.5454-5459, 1995.

[10] M. G. Kong, "Mode-selective amplification in a waveguide free electron laser with a two-sectioned wiggler", IEEE Trans. Plasma Sci. vol.26, pp.85-91, (1998).

[11] T. I. Smith, J. M. J. Madey, L. R. Elias, and D. A. G. Deacon, "Reducing the sensitivity of a free-electron laser to electron energy", J. Appl. Phys., vol.50, pp.4580-4583, 1979.

[12] L. K. Grover and R. H. Pantell, "An upper limit to the gainenergy acceptance produce of the free electron laser", IEEE J. Quan. Electron., vol.22, pp.789-792, 1986.

[13] M. G. Kong and A. Vourdas, "A new free electron laser interaction mechanism based on a quasiperiodic wiggler", Proceedings of the 25th IEEE International Conference on Plasma Science, IEEE Publication 98CH36221, p.195, 1998. 